\begin{tabular}{|c|c|}
\hline Title & Effect of Structure Heterogeneity on Mechanical Performance of Physical Polyampholytes Hydrogels \\
\hline Author(s) & $\begin{array}{l}\text { Cui, Kunpeng; Y e, Y a Nan; Sun, T ao Lin; Chen, Liang; Li, X ueyu; Kurokawa, Takay uki; Nakajima, T asuku; } \\
\text { Nonoyama, Takayuki; Gong, Jian Ping }\end{array}$ \\
\hline Citation & $\begin{array}{l}\text { Macromolecules, 52(19), 7369-7378 } \\
\text { https://doi.org/10.1021/acs.macromol.9b01676 }\end{array}$ \\
\hline Issue Date & $2019-10-08$ \\
\hline Doc URL & http:/hdl.handle.net/2115/79491 \\
\hline Rights & $\begin{array}{l}\text { This document is the A ccepted Manuscript version of a Published Work that appeared in final form in Macromolecules, } \\
\text { copyright } @ \text { A A merican Chemical Society after peer review and technical editing by the publisher. To access the final } \\
\text { edited and published work see https:/pubs.acs.org/doi/10.1021/acs.macromol.9b01676. }\end{array}$ \\
\hline Tyре & article (author version) \\
\hline Additional Information & There are other files related to this item in HUSCAP. Check the above URL. \\
\hline File Information & Macromolecules_52-19_7369-7378.pdf \\
\hline
\end{tabular}

Instructions for use 


\section{Effect of Structure Heterogeneity on Mechanical Performance of Physical Polyampholytes Hydrogels}

Kunpeng Cui ${ }^{1}$, Ya Nan Ye,3, Tao Lin Sun ${ }^{2,3,4}$, Liang Chen ${ }^{5}$, Xueyu $\mathrm{Li}^{3}$, Takayuki Kurokawa $^{2,3}$, Tasuku Nakajima ${ }^{1,2,3}$, Takayuki Nonoyama ${ }^{2,3}$, Jian Ping Gong ${ }^{1,2,3^{*}}$

${ }^{1}$ Institute for Chemical Reaction Design and Discovery (WPI-ICRD), Hokkaido University, Sapporo 001-0021, Japan

${ }^{2}$ Faculty of Advanced Life Science, Hokkaido University, Sapporo 060-0810, Japan

${ }^{3}$ Soft Matter GI-CoRE, Hokkaido University, Sapporo 001-0021, Japan

${ }^{4}$ South China Advanced Institute for Soft Matter Science and Technology, South China University of Technology, Guangzhou 510640, China

${ }^{5}$ Graduate School of Life Science, Hokkaido University, Sapporo 060-0810, Japan

*Corresponding author: gong@sci.hokudai.ac.jp

\section{ABSTRACT}

Recent studies reported multiscale structure in tough and self-healing hydrogels containing physical associations. For example, a type of tough and self-healing hydrogels from charged balanced polyampholytes (PA) have a meso-scale bicontinuous double network structure with structural length around $400 \mathrm{~nm}$. This meso-scale network structure plays an essential role in the multistep rupture process, which leads to the high toughness of PA hydrogels. In this work, by using an osmotic stress method, we symmetrically studied how the relative strength of soft and hard networks and the strength of ionic bonds influence the property of PA gels. We found that, increasing osmotic stress of the bath solution triggers the structure transition from bicontinuous double network structure to a homogeneous structure, which drives the concurrently opaque-transparent transition in optical property, 
and viscoelastic-glassy transition in mechanical behavior. The gels around the structural transition point were found to possess both high toughness (fracture energy of $7200 \mathrm{~J} \mathrm{~m}^{-2}$ ) and high stiffness (Young's modulus of 12.9 MPa), which is a synergy of soft network and hard network of the bicontinuous structure. Our work not only provides an approach to tune the structure and property of physical hydrogels through tuning physical association, but also gives a demo to investigate their relationships, yet another step forward, gives inspiration to design new type of tough and self-healing materials around the structural transition point.

\section{INTRODUCTION}

Hydrogels composed of three-dimensional polymer network and abundance of water have attracted considerable attention due to their prime importance in many fields, such like stimuli response ${ }^{1-3}$, drug delivery ${ }^{4,5}$ and tissue engineering ${ }^{6}$. However, the applications of hydrogels are usually restricted seriously by their mechanical weakness ${ }^{7-9}$. Since 2000 , substantial efforts have been devoted to develop strong and tough hydrogels ${ }^{10-14}$, among them the double-network (DN) gels, by incorporating a stiff and brittle network as sacrificial covalent bonds into a soft and ductile network matrix, stands out as one of the most tough hydrogels ${ }^{15-19}$. Inspired by the sacrificial bond concept used in DN gels, various tough and strong physical hydrogels with partially or even completely self-healing ability were successfully developed, by using reversible physical bonds as sacrificial bonds ${ }^{20-25}$. For example, the physical hydrogels from polyampholytes (PA gels) with ionic interactions as sacrificial bonds, possess not only high toughness and high fatigue resistance, but also $100 \%$ self-recovery and a very broad relaxation spectrum. ${ }^{21,26,27}$ 
Despite great success on creating tough and self-healing hydrogels through reversible physical bonds, the fundamental basis, the structure and the toughening mechanism of these gels, remains poorly understood. These physical hydrogels have been described by a simple dichotomic molecular picture of the elastic network crosslinked by weak bonds and strong bonds, whereupon the weak bonds can break to dissipate energy and reform to impart selfhealing, and strong bonds can maintain the integrity of the gels over a much longer timescale. Recent studies reported the large scale heterogeneous structure in these gels with structural length in tens or hundreds nanometers scale, like PA system in water ${ }^{21}$, polyacrylamide system in a mixture of good and poor solvents ${ }^{28}$ or poly(N-isopropylacrylamide) system above the critical temperature ${ }^{29}$, suggesting that the dichotomic molecular picture is too oversimplified. Especially, our recent study by combing atomic force microscopy (AFM) and time-resolved small-angle X-ray scattering (SAXS) suggests that the PA hydrogels have a multiscale structure, and the toughening results from a synergistic effect involving multiscale energy dissipation. ${ }^{30}$

PA gels has a hierarchical structure of several length scale: at the monomer scale, the positive and negative charges associate to form ionic interactions; at the nanoscale, the charged chains aggregate into soft and hard regions, which differ in modulus and polymer density; in the mesoscale, the soft and hard regions form a bicontinuous double network structure. This multiscale structure leads a multiscale energy dissipation during deformation. ${ }^{30}$ Upon loading, the microscopic deformation of bicontinuous networks is perfectly affine up to a quite large macroscopic uniaxial tensile deformation range, followed by a wide nonaffine deformation range. In affine deformation regime, the breaking of ionic 
bonds in monomeric scale occurs to induce unfolding of the globular chains, permitting a large mechanical hysteresis, but the samples are fully self-recoverable due to self-healing effect of the dynamic physical bonds. In the nonaffine deformation regime, the hard network ruptures due to the high stiffness and the samples exhibit permanent softening while their shape are fully recoverable, due to elasticity of the soft network. In nonaffine regime, the soft network effectively transfers stress and reduces stress concentration, preventing the catastrophic propagation of hard network rupture. Finally, the rupturing of soft network leads to the global failure of sample. Such meso-scale double network structure prevents the catastrophic propagation of hard network rupture, leading to a large deformability of the materials before global failure. This multi-scale rupture process dissipates a significant amount of energy to give a high toughness of gels.

Given the above picture, the mechanical properties of the PA gels should be correlated to the strength of ionic interactions, and the relative strength of soft and hard networks. The former is relevant for the energy dissipation and viscoelasticity of gels. The latter is relevant for stress transfer and reduction of stress concentration when the hard network rupturing. In this work, we aim to study how the strength of ionic interaction, and the relative strength of soft and hard networks influence the properties of PA hydrogels. For this purpose, an osmotic stress method is adopted by using the neutral and biocompatible poly(ethylene glycol) (PEG) solutions to tune the osmotic pressure of the bath solution ${ }^{31,32}$. We start with PA gel equilibrated in pure water. The bicontinuous mesoscale double network structure formed in PA gels is the result of thermo equilibrium in water. When the bath solution is changed from water to PEG solution, an osmotic pressure is exerted on the PA gel 
from the solution. As a result, the gel is dehydrated to reach a new equilibrium state. As the ionic associative energy increases with polymer volume fraction ${ }^{21,33}$, the strength of ionic interaction is tuned by tuning osmotic stress without changing the topological chain structure of gels. Since the dehydration abilities of the dense, hard network region and the sparse, soft network region are different, the relative strength of the two networks is expected to change with the PEG concentration.

In this work, the effects of relative strength of soft and hard networks, and the strength of ionic interaction on mechanical property of PA hydrogels were systematically studied with the osmotic stress method. Our results show that, by increasing osmotic stress of the bath solution, the contrast between soft and hard networks decreases and eventually disappears, that is, the gel transforms from heterogeneous structure to homogeneous structure. This heterogeneous-homogeneous transition in structure is concomitant with an opaquetransparent transition in optical property. At a given observation time, the gels with a heterogeneous structure is ductile and with a homogeneous structure become glassy, while the gels near the structural transition point possess the maximum toughness. This maximum toughness is a synergy of increased strength of ionic bonds to dissipate energy and reduced deformability due to structural homogenization.

\section{EXPERIMENTS}

Materials. All the materials, including anionic monomer p-styrenesulfonate (NaSS), cationic monomer 3-(methacryloylamino) propyl-trimethylammonium chloride (MPTC), photoinitiator $\alpha$-ketoglutaric acid ( $\alpha$-keto), and PEG with weight-average molecular weight 
$M_{\mathrm{w}}$ of $2 \times 10^{4} \mathrm{~g} / \mathrm{mol}$ were pursed from Wako Prue Chemical Industries, Ltd (Japan) and used as received. Deionized water was used in all of the experiments.

Synthesis of PA hydrogels. The charge balanced P(NaSS-co-MPTC) hydrogels were synthesized with a one-step random copolymerization procedure, which has been described in detail in our previous work. ${ }^{21,34}$ In brief, a mixed aqueous solution containing NaSS, MPTC and $\alpha$-keto was injected into a sandwich like sample cell (thickness: $2 \mathrm{~mm}$ ) and irradiated with UV light (wavelength of $365 \mathrm{~nm}$ ) for 11 hours under argon atmosphere. The total monomer concentration $C_{\mathrm{m}}$ is $2.1 \mathrm{M}$, the molar ratio of NaSS : MPTC is $0.525: 0.475$, and the concentration of $\alpha$-keto is $0.25 \mathrm{~mol} \%$ relative to $C_{\mathrm{m}}$. The PA synthesized at this formulation has balanced charges. After polymerization, the obtained gels (as-prepared gels) were immersed into large amount of water longer than one week at room temperature to remove counterions and residual chemicals and to reach equilibrium. In water, the sample shrank isotopically, and the thickness changed from $2.0 \mathrm{~mm}$ to about $1.9 \mathrm{~mm}$.

Preparation of PA hydrogels with different polymer volume fraction. The osmotic pressure of the bath solution is tuned roughly in a range from 0 to $10 \mathrm{MPa}$ by using PEG aqueous solutions with weight percentage from $0 \mathrm{wt} \%$ to $60 \mathrm{wt} \%{ }^{31,32}$. There is no preferential interaction between PEG and PA, and the molecular weight of PEG is large enough that the diffusion of PEG into the PA gel is negligible. The water equilibrated hydrogels were immersed into various PEG solutions to re-equilibrate for one week at room temperature. After re-equilibration, the water weight percentage of gel (or water content) $c_{w}$ was measured by the weight change upon drying at $120^{\circ} \mathrm{C}$ with an electronic moisture balance device (Figure S1a, Supporting Information). The polymer volume fraction $\left(\phi_{\mathrm{p}}\right)$ of 
hydrogels was determined by the equation $\phi_{\mathrm{p}}^{-1}=1+\left(\frac{c_{w}}{1-c_{w}}\right) \frac{\rho_{\mathrm{p}}}{\rho_{\mathrm{w}}}$, where $\rho_{\mathrm{p}}=1.19 \pm$ $0.02 \mathrm{~g} \mathrm{~cm}^{-3}$ and $\rho_{\mathrm{w}}=0.98 \mathrm{~g} \mathrm{~cm}^{-3}$ are the density of PA and water at room temperature, respectively. ${ }^{35}$

Optical transmittance test. The transmittance of the gels was characterized by a Shimadzu UV spectrophotometer with wavelength from 500 to $700 \mathrm{~nm}$ at room temperature of $24{ }^{\circ} \mathrm{C}$ (Figure S1b, Supporting Information). The experiment was conducted in PEO solution at room temperature. PEG solutions without gel were also measured as background to correct the transmittance of gels.

X-ray scattering measurement. The SAXS measurement was performed at BL16B beamline of Shanghai Synchrotron Radiation Facility (SSRF, China). The X-ray energy used was $10 \mathrm{keV}$ and the distance between sample and detector was $5370 \mathrm{~mm}$. A Mar $165 \mathrm{CCD}$ detector $(2048 \times 2048$ pixels with pixel size of $80 \mu \mathrm{m})$ was used to collect two-dimensional (2D) scattering patterns with data acquisition time of $20 \mathrm{~s}$ per frame. Ultra-small-angle Xray scattering (USAXS) measurement was conducted at BL20XU beamline of Spring-8 (JASRI, Japan). The X-ray energy was $23 \mathrm{keV}$ and the distance between sample and detector was $160.5 \mathrm{~m}$. A Pilatus $100 \mathrm{~K}$ detector $(487 \times 195$ pixels with pixel size of $172 \mu \mathrm{m})$ was used with data acquisition time of $60 \mathrm{~s}$ per frame. In all X-ray scattering measurements, gels were measured at room temperature and covered with Kapton film to prevent possible drying. SAXS and USAXS data were analyzed with Fit 2D software from European Synchrotron Radiation Facility ${ }^{36}$ and corrected for detector spatial distortion, X-ray beam fluctuation and background scattering. 
Rheology test. The rheological measurement was conducted with a parallel-plate geometry using an ARES-G2 rheometer (TA instruments, USA). A frequency sweep varied from 0.1 to $100 \mathrm{rad} / \mathrm{s}$ was performed with a constant strain of $0.1 \%$. Disc-shape samples with a diameter of $15 \mathrm{~mm}$ and a thickness of 1.6-1.9 $\mathrm{mm}$ were adhered to the parallel plates with superglue and surround by PEG solution or paraffin oil during measurement. The frequency sweep experiments were conducted at different temperatures from 0 to $95{ }^{\circ} \mathrm{C}$ with per $8{ }^{\circ} \mathrm{C} \mathrm{a}$ step. Before measurement, the sample was held at the pre-set temperature for $300 \mathrm{~s}$ to reach equilibrium, and the auto-strain function as well as manual gap adjustment were used to minimize compression on the sample.

Mechanical test. Two sets of uniaxial tensile tests were carried out, one is at room temperature, another is at high temperature $\left(52^{\circ} \mathrm{C}\right)$. The room temperature test was performed in an Instron 5965 tensile tester (Instron Co.), and the high temperature test was performed in a Tensilon RTC-1310A tester (Orientec Co.). Samples were cut into a dumbbell-like shape with the JIS K 6261-7 standard (12 mm in gauge length and $2 \mathrm{~mm}$ in width). The tensile speed was fixed at $100 \mathrm{~mm} / \mathrm{min}$, which gives a nominal strain rate of 0.14 $\mathrm{s}^{-1}$. The nominal strain rate is defined as the ratio of stretch speed to gauge length. The stress $\sigma$ is defined as the force divided by the initial cross-section area of sample and the Young's modulus $E$ is calculated from the slop of initial linear region of stress-strain curve. The yielding stress $\sigma_{\mathrm{y}}$ and yielding strain $\varepsilon_{\mathrm{y}}$ are calculated from the point where the slop of stressstrain curve is zero. The fracture stress $\sigma_{\mathrm{b}}$ and fracture strain $\varepsilon_{\mathrm{b}}$ are defined as the point where breaking occurs. The work of extension at fracture $W_{\text {extf }}$ is calculated from the area under the stress-strain plot up to fracture. A loading-unloading test was performed at room 
temperature with the same sample size and stretch speed as uniaxial tensile test. The maximum strain of loading process was set as 3.0 and the unloading speed was the same as the loading speed. Single-edge notch test $\mathrm{t}^{37,38}$ was performed at room temperature to obtain fracture energy. In the single-edge notch test, rectangular samples with length of $20 \mathrm{~mm}$, width of $10 \mathrm{~mm}$, and without notch or with notch length $c$ of $1 \mathrm{~mm}$ were used. The stretch speed was $100 \mathrm{~mm} / \mathrm{min}$. The fracture energy $\Gamma$ was estimated by the equation $\Gamma=$

$2 \frac{3}{\sqrt{\lambda_{\mathrm{c}}}} c W\left(\lambda_{\mathrm{c}}\right)$. Here $W\left(\lambda_{\mathrm{c}}\right)=\int_{1}^{\lambda_{c}} \sigma_{\mathrm{un}-\text { notched }} d \lambda$ is the stored energy density ${ }^{37}$ of an unnotched sample which can be estimated from the yellow area in Figure $6 b$ or $6 c, \lambda_{c}$ is the critical stretch ratio where crack propagation starts for the notched sample. In all mechanical measurements, samples were surround by PEG solution or paraffin oil to prevent possible drying. In each measurement, samples were measured at least three times to make an average and to calculate the error bar.

\section{RESULTS AND DISCUSSION}

\section{Optical property.}

The polyampholytes obtained in the as-prepared state are topologically entangled with each other and each polymer chain possesses oppositely charged ionic groups randomly distributed along the chain backbone. By dialysis of small counterions in pure water, ionic associations between opposite charges on the polymer chains, both intrachain and interchain, are switched on, forming a bicontinuous network structure, consisting a soft network (with low modulus) and a hard network (with high modulus), of $\sim 100 \mathrm{~nm}$ in scale. ${ }^{30}$ Figure 1a presents the pictures of PA hydrogels after re-equilibration in PEG solutions with different 
PEG concentrations. The initial sizes of the gels are the same with a $10 \mathrm{~mm}$ diameter and a $1.9 \mathrm{~mm}$ thickness. After being immersed in PEG solution, the gels shrink by osmotic stress. The volume of gels decreases about $50 \%$, while the corresponding polymer volume fraction $\phi_{\mathrm{p}}$ increases about $120 \%$, in the studied $C_{\mathrm{PEG}}$ ranging from $0 \mathrm{wt} \%$ to $60 \mathrm{wt} \%$ (Figure 1c). When $C_{\mathrm{PEG}}$ is smaller than $20 \mathrm{wt} \%$, the gels are opaque, as demonstrated by the photo pictures in Figure 1a and by the almost zero transmittance in Figure $1 \mathrm{~b}$. While when $C_{\mathrm{PEG}}$ reaches $20 \mathrm{wt} \%$, the gel becomes translucent, thus the background lattice becomes visible. The transparency of gels increases rapidly for $C_{\mathrm{PEG}}>20 \mathrm{wt} \%$ and almost transparent gels can be obtained when $C_{\mathrm{PEG}}$ is larger than $40 \mathrm{wt} \%$. This result shows that an opaquetransparent transition occurs in PA hydrogels by dehydration at $C_{\mathrm{PEG}}$ around $30 \mathrm{wt} \%$, as shown by dotted red line in Figure $1 \mathrm{~b}$ as a boundary. Similar opaque-transparent transition has also been reported in natural soft matter such as egg white or vitreous bodies of fish eyeballs during dehydration, in which strong physical associations exist. ${ }^{39}$ 

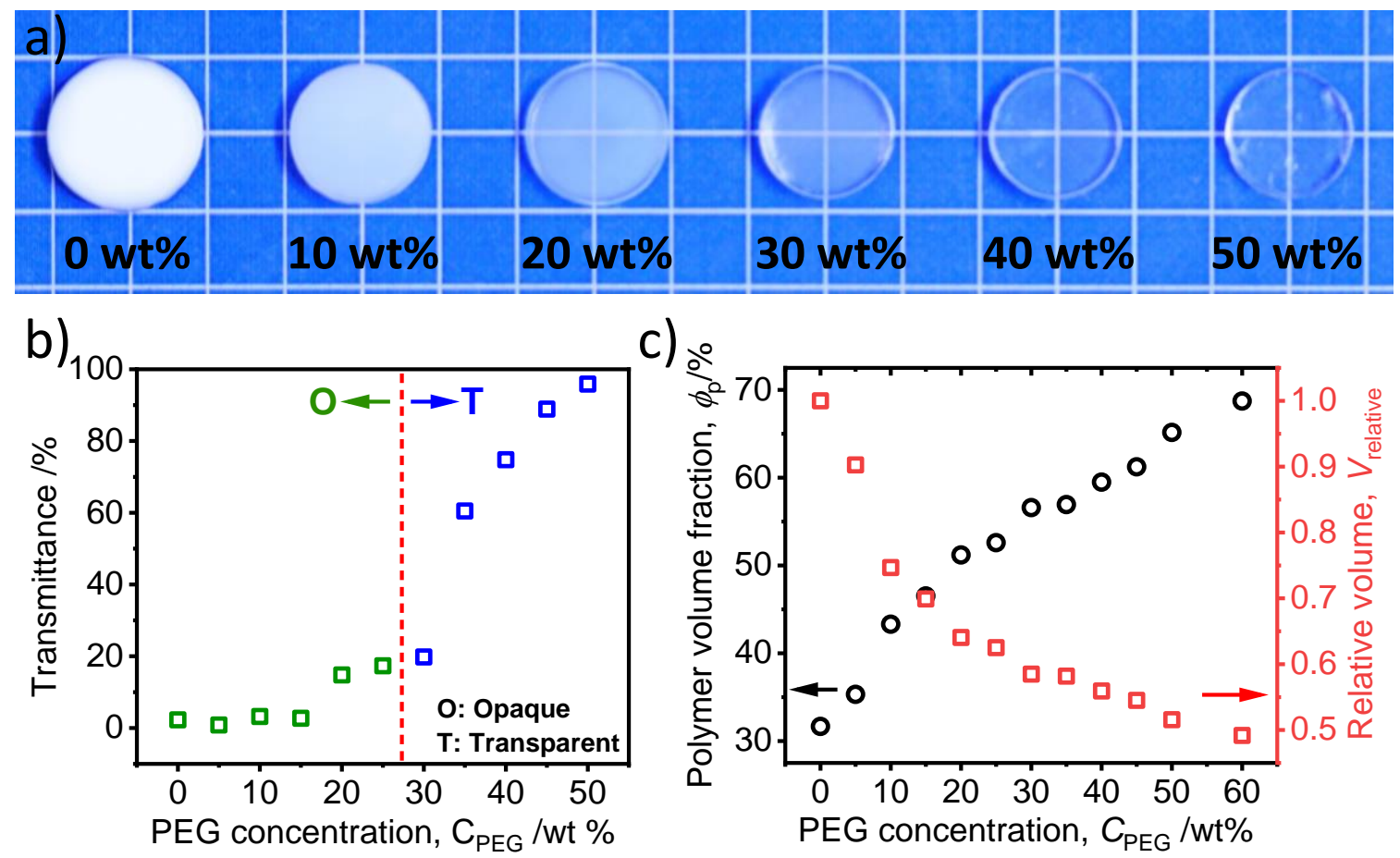

C) $\stackrel{0}{\circ}$

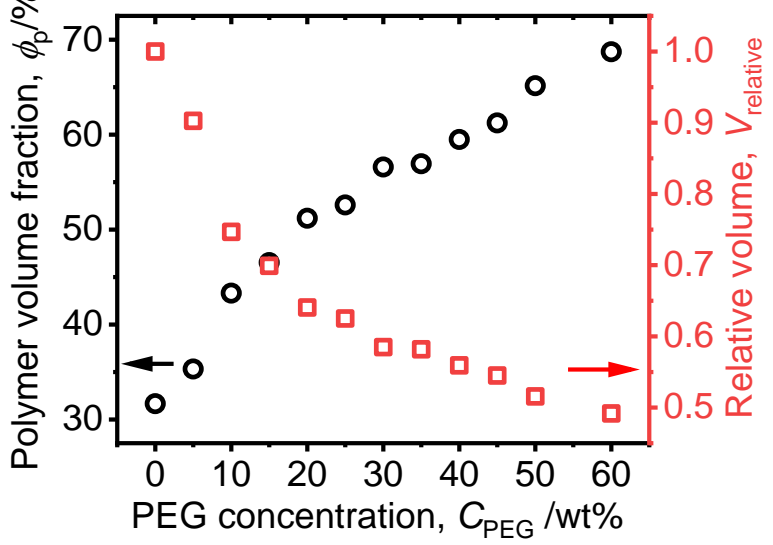

Figure 1. (a) Photographs of re-equilibrated PA hydrogels in different PEG aqueous solutions. The numbers in (a) are the PEG weight fraction of PEG aqueous solutions. The mesh size of background lattice is $5 \mathrm{~mm}$. (b) The average transmittance of wavelength from 500 to 700 $\mathrm{nm}$, and (c) the polymer volume fraction as well as the relative volume of PA hydrogels as a function of PEG concentration. The relative volume $V_{\text {relative }}$ is defined as the volume of the gel in PEG solution compared to that in pure water.

\section{Structural change.}

To explore structural change behind the opaque-transparent transition in optical property, we combined USAXS and SAXS to study the structure of PA hydrogels at different $\phi_{\mathrm{p}}$. The gels with relatively low $\phi_{\mathrm{p}}$ (in low $C_{\mathrm{PEG}}$ solutions) are opaque, suggesting the heterogeneity in structure with structural length around or above the wavelength of visible light. Our previous study has demonstrated that this heterogeneity is due to the formation of bicontinuous network structure. ${ }^{30}$ Starting from the bicontinuous network structure, the opaque-transparent transition by increasing $\phi_{\mathrm{p}}$ may be caused by two reasons. One is that, 
the bicontinuous network structure transforms into homogeneous structure. Another is that, the structure of PA gel is still heterogeneous by increasing $\phi_{\mathrm{p}}$, but the structure length decreases to a value far smaller than the wavelength of visible light. The USAXS with $q$ ranging from 0.0037 to $0.035 \mathrm{~nm}^{-1}$ can probe structure length from 1697 to $179 \mathrm{~nm}$, which covers structure change in the scale of visible light region and beyond. While the SAXS with $q$ ranging from 0.027 to $0.70 \mathrm{~nm}^{-1}$ can capture structural length from 233 to $9 \mathrm{~nm}$, which is expected to verify whether the structure length of bicontinuous structure decrease to a much smaller size. Here $q=4 \pi \sin \theta / \lambda$ is the modulus of scattering vector, $2 \theta$ is the scattering angle and $\lambda$ is the $\mathrm{X}$-ray wavelength.
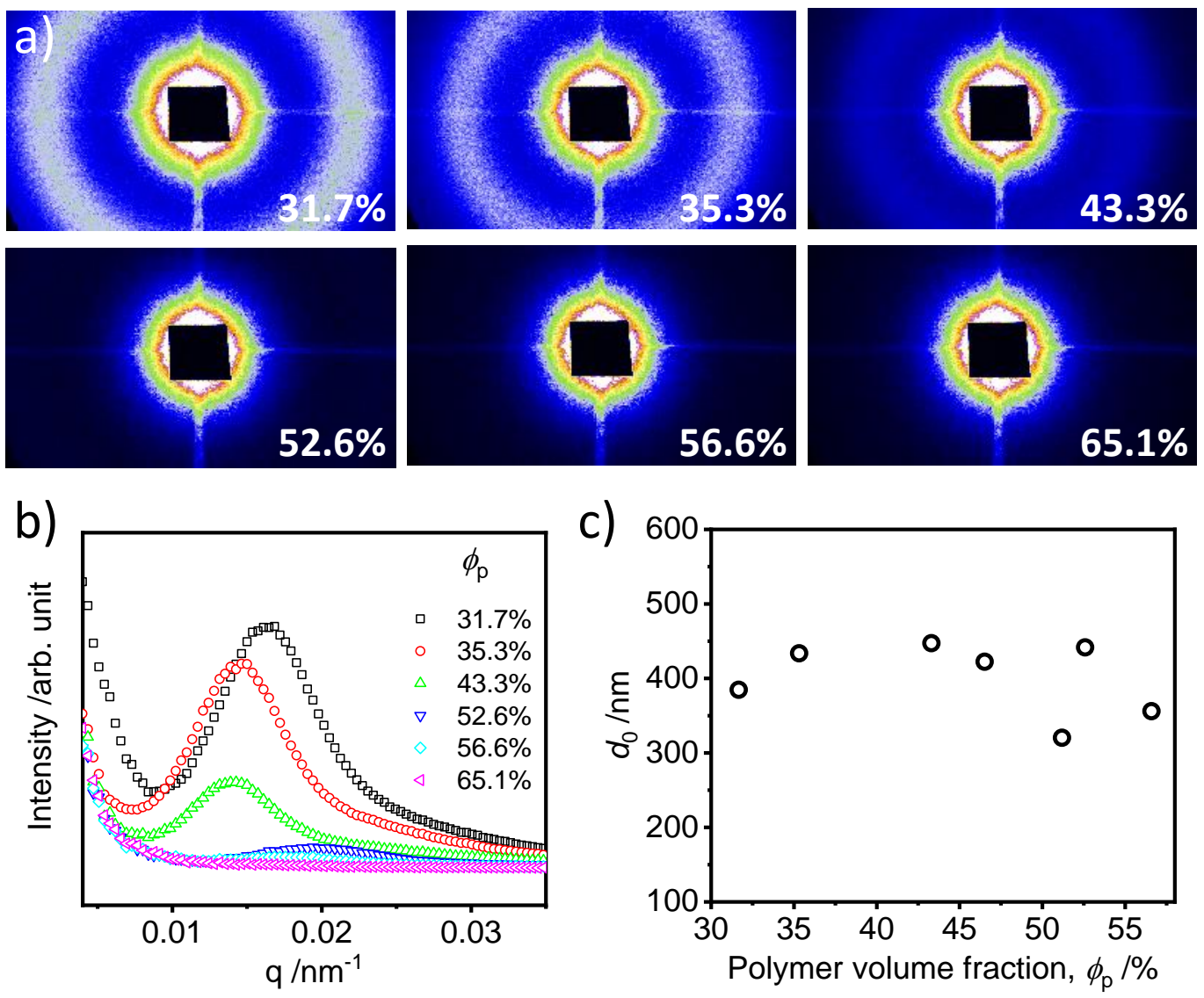
Figure 2. (a) 2D USAXS patterns of PA hydrogels at different polymer volume fraction $\phi_{\mathrm{p}}$ and (b) the corresponding 1D intensity profiles. (c) The characteristic structural length $d_{0}$ as a function of polymer volume fraction $\phi_{\mathrm{p}}$.

Figure 2a reports the 2D USAXS pattern of PA gels at different $\phi_{\mathrm{p}}$. For $\phi_{\mathrm{p}} \leq 52.6 \%$, an isotropic pattern with a circular scattering ring appears, demonstrating the heterogeneous structure of gels. Note that the streak scattering around beam stop in 2D USAXS patterns is due to the background scattering of beamline. The scattering ring in $2 \mathrm{D}$ pattern gives rise to a correlation peak between $q=0.01$ and $0.02 \mathrm{~nm}^{-1}$ in the 1D scattering intensity profile (Figure $2 \mathrm{~b}$ ), corresponding to a characteristic structural length (the spacing between adjacent soft regions or adjacent hard regions) $d_{0}$ about $400 \mathrm{~nm}$ (Figure $2 \mathrm{c}$ ) that accounts for the opaque appearance of gels, in consistent with the previous report. ${ }^{30} d_{0}$ is calculated from the equation $d_{0}=2 \pi / q_{\text {peak }}$, where $q_{\text {peak }}$ is the peak position in the $1 \mathrm{D}$ scattering intensity profile. The intensity of scattering ring in 2D pattern (Figure 2a) and the corresponding correlation peak in 1D profile (Figure 2b) decrease by increasing $\phi_{\mathrm{p}}$ for $\phi_{\mathrm{p}} \leq 52.6 \%$, suggesting the degree of heterogeneity decrease with increasing $\phi_{\mathrm{p}}$, while the value of $d_{0}$ hardly changes with increasing $\phi_{\mathrm{p}}$ (Figure $2 \mathrm{c}$ ). When $\phi_{\mathrm{p}}>52.6 \%$, no visible scattering ring can be observed in the 2D scattering pattern and typical amorphous scattering or very faint peak appears in the 1D scattering intensity profile, indicating the gels become homogeneous in the detectable structural length of USAXS. 


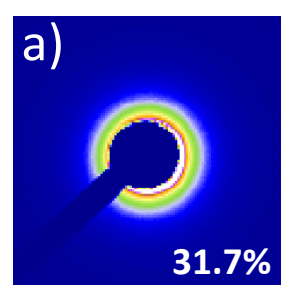

b)
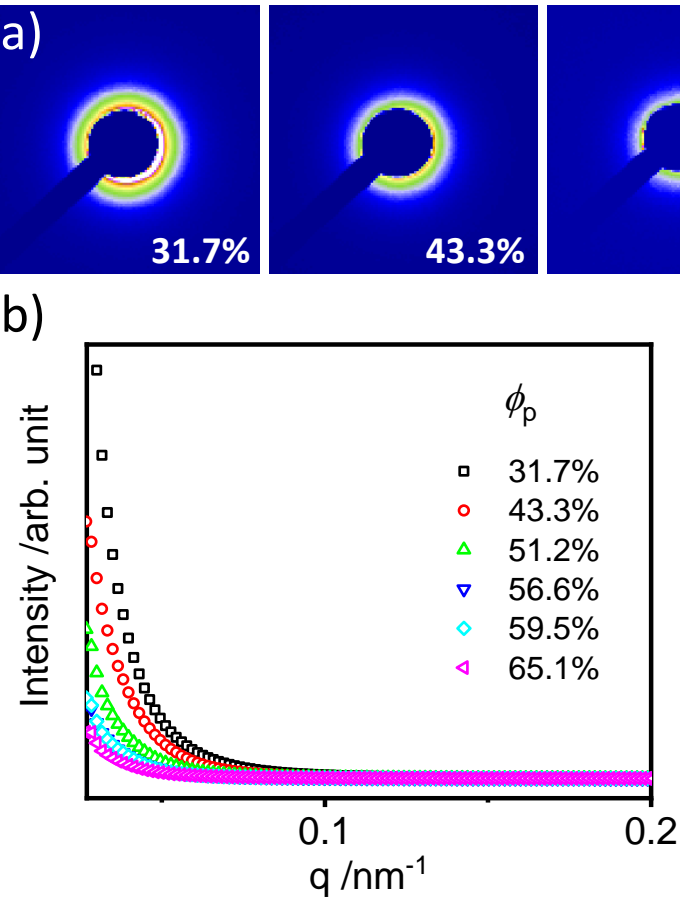
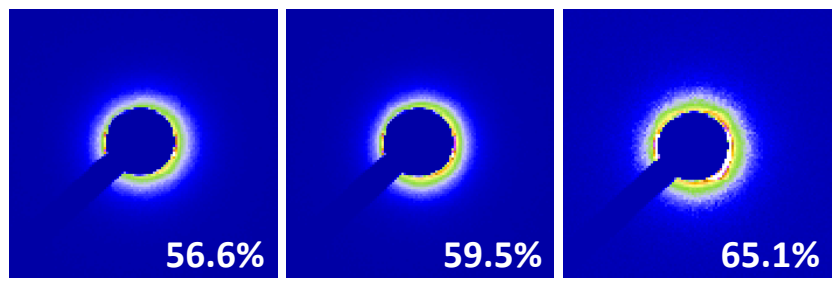

c)

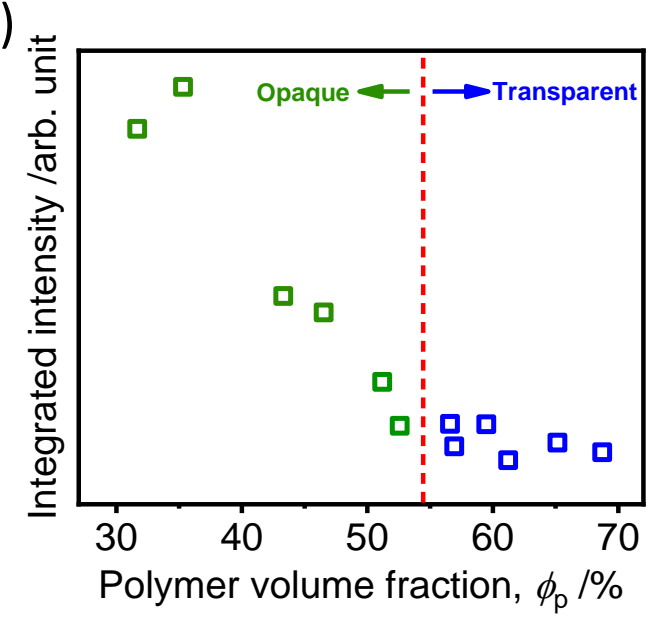

Figure 3. (a) 2D SAXS patterns of PA hydrogels at different polymer volume fraction $\phi_{\mathrm{p}}$ and (b) the corresponding 1D scattering intensity profiles. (c) The integrated intensity as a function of $\phi_{\mathrm{p}}$. To highlight the intensity change in the 1D plot, data only at $q$ ranging from 0.027 to $0.20 \mathrm{~nm}^{-1}$ are present in (b).

To examine the second possibility, i.e., whether the opaque-transparent transition is due to that the structure length of bicontinuous structure decreases to a much smaller size, SAXS was conducted and the obtained results are shown in Figure 3. In all studied $\phi_{\mathrm{p}}$, no scattering ring presents in the 2D SAXS patterns (Figure 3a) and no correlation peak arises in the $1 \mathrm{D}$ scattering intensity profiles (Figure $3 \mathrm{~b}$ ), demonstrating the absence of bicontinuous structure with structural length between 233 to $9 \mathrm{~nm}$. Thus, the opaque-transparent transition is not due to the decrease in the structure length of bicontinuous structure. Meanwhile, the scattering intensity decreases by increasing $\phi_{\mathrm{p}}$ in the whole $q$ range investigated (Figure $3 b$ ), which is consistent with the results reported by Li et al. ${ }^{40}$ To analyze the scattering intensity 
change quantitatively, the 1D intensity profile was integrated in the whole studied $q$ range and was plotted as a function of $\phi_{\mathrm{p}}$ (Figure 3c). The scattering in SAXS can be considered as the high- $q$ scattering regime of the USAXS (Figure S2, Supporting Information), which can be used to reflect the interface between soft and hard regions of bicontinuous structure. ${ }^{41}$ As shown in Figure 3c, the integrated intensity decays dramatically for $\phi_{\mathrm{p}} \leq 52.6 \%$, suggesting that the difference between two regions, i.e., the contrast, decreases by increasing $\phi_{\mathrm{p}}$, in agreement with USAXS results. Then the integrated intensity reaches a plateau, further demonstrating that the structure of PA become homogeneous for $\phi_{\mathrm{p}}>52.6 \%$.

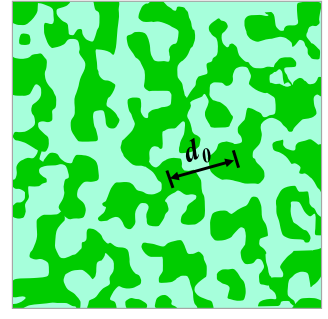

a. Heterogeneous

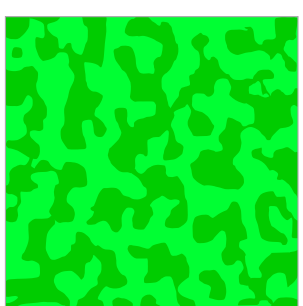

b. Transition point

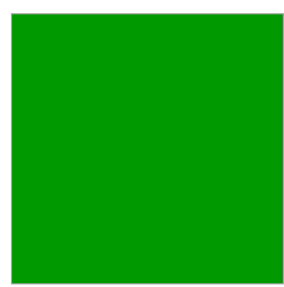

c. Homogeneous

\section{Low Osmotic stress High}

Polymer dense region

Polymer sparse region

Figure 4. Schematic of structure evolution of PA hydrogels by changing osmotic stress from low to high.

Combing USAXS and SAXS results, one safe conclusion can be drawn is that, the bicontinuous structure with $d_{0}$ around $400 \mathrm{~nm}$ of PA gels transforms into a homogeneous structure by increasing $\phi_{\mathrm{p}}$ (Figure 4). The boundary defined by heterogeneous-homogeneous transition in structure corresponds to the boundary defined by opaque-transparent transition in optical property exactly, suggesting that the heterogeneous-homogeneous transition is the 
structural origin of the transmittance change in optical behavior. The gel starts with a bicontinuous network structure (Figure 4a), increasing the osmotic stress of PEG solution leads to the condensation of polymer. According to our previous work, the Young's modulus of the soft network and the hard network are 0.3 MPa and 1.9 MPa, respectively, measured by atomic force microscopy with a scan rate of $0.5 \mathrm{kHz} \cdot{ }^{30}$ Due to the difference in stiffness, the polymers in soft network (polymer sparse region) is much easier to condense than that of hard network (polymer dense region) under osmotic stress. Thereby the contrast between soft and hard networks decreases with increasing osmotic stress, as demonstrated by the USAXS results in Figure 2 and the SAXS results in Figure 3. When the osmotic stress or $\phi_{\mathrm{p}}$ is larger a threshold ( $\phi_{\mathrm{p}}=52.6 \%$ for PA in this work), the "polymer sparse region" becomes dense enough as "polymer dense region" to form a homogeneous structure (Figure 4c). Here one question needs to explain is that why $d_{0}$ seems do not change with $\phi_{\mathrm{p}}$ for $\phi_{\mathrm{p}} \leq 52.6 \%$ (Figure 2c). Assuming the bicontinuous structure undergoes homogeneous deformation

during dehydration, $d_{0}$ should be proportional to $1 /\left(\phi_{\mathrm{p}}^{1 / 3}\right)$. An increase in polymer volume fraction from $31.7 \%$ to $52.6 \%$ only lead to $15 \%$ reduction in $d_{0}$, which may be smeared by the deviation of different samples.

\section{Viscoelastic-glassy transition.}

The relationship between structure and mechanical property was investigated by performing uniaxial tensile test. As demonstrated by the rheological data in Figure S3 (Supporting Information) as well as in our previous work ${ }^{21}$, PA hydrogels with bicontinuous structure are strongly viscoelastic with a very broad dynamic relaxation spectra and the 
mechanical property strongly depends on the imposed strain rate. Here a specific observation time or observation strain rate (nominal strain rate $0.14 \mathrm{~s}^{-1}$ ) was chosen, which is the same as that in our previous work ${ }^{34,35}$. Figure 5 a presents tensile stress-strain curves at four selected polymer volume fraction $\phi_{\mathrm{p}}$ at room temperature. For $\phi_{\mathrm{p}} \leq 52.6 \%$, the PA hydrogels are tough and ductile. Taking sample with $\phi_{\mathrm{p}}=31.7 \%$ as an example, the Young's modulus $E$, fracture stress $\sigma_{\mathrm{b}}$ and fracture strain $\varepsilon_{\mathrm{b}}$ can reach 1.7 $\mathrm{MPa}, 1.9 \mathrm{MPa}$ and 9.4, respectively. When $\phi_{\mathrm{p}}>52.6 \%$, the gels become glassy-like, manifesting with a clear yielding like in glassy $^{42}$ or semicrystalline ${ }^{43}$ polymers. After yielding, a stress plateau appears, followed by a stress upturn. Meanwhile, both the Young's modulus and fracture stress are significantly increased, accompanying with a decrease in fracture strain. The fracture strain decreases to 1.8 when $\phi_{\mathrm{p}}=65.1 \%$, which is rather brittle compared to the sample with $\phi_{\mathrm{p}}=31.7 \%$ $\left(\varepsilon_{\mathrm{b}}=9.4\right)$. In our previous study ${ }^{30}$, real time SAXS result suggests that the hard phase starts to rupture around a strain of 3, which is reasonably consistent with the fracture strain of homogeneous glassy samples at $\phi_{\mathrm{p}}>52.6 \%$.

The viscoelastic-glassy transition in tensile behavior is compared with the linear rheological behavior. The nominal strain rate $\dot{\varepsilon}=0.14 \mathrm{~s}^{-1}$ in tensile test corresponds to a frequency $\omega$ of $0.14 \mathrm{rad} / \mathrm{s}$ in rheology test. In case of gel with $\phi_{\mathrm{p}}=31.7 \%$, the loss factor $\tan \delta$ is 0.74 at $\omega=0.14 \mathrm{rad} / \mathrm{s}$ and the storage modulus $G^{\prime}$ as well as loss modulus $G^{\prime \prime}$ are strongly frequency dependent around $0.14 \mathrm{rad} / \mathrm{s}$ (Figure S3a, Supporting Information). Thus, this sample is observed in its highly viscoelastic regime. While in case of gel with $\phi_{\mathrm{p}}=$ $65.1 \%, \tan \delta$ is only 0.15 at $\omega=0.14 \mathrm{rad} / \mathrm{s}$, and $G^{\prime}$ has very weak and $G^{\prime \prime}$ has almost no 
frequency dependency around $0.14 \mathrm{rad} / \mathrm{s}$ (Figure S3b, Supporting Information). This is the typical rheological behavior of glassy polymers, which explains well why this sample is glassy-like.

a)

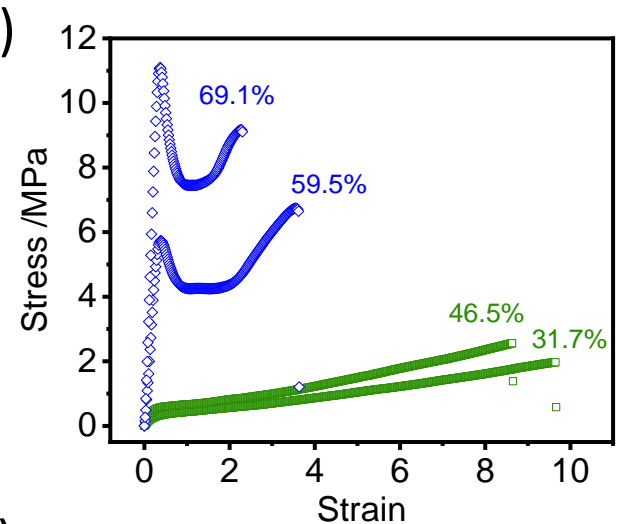

c)

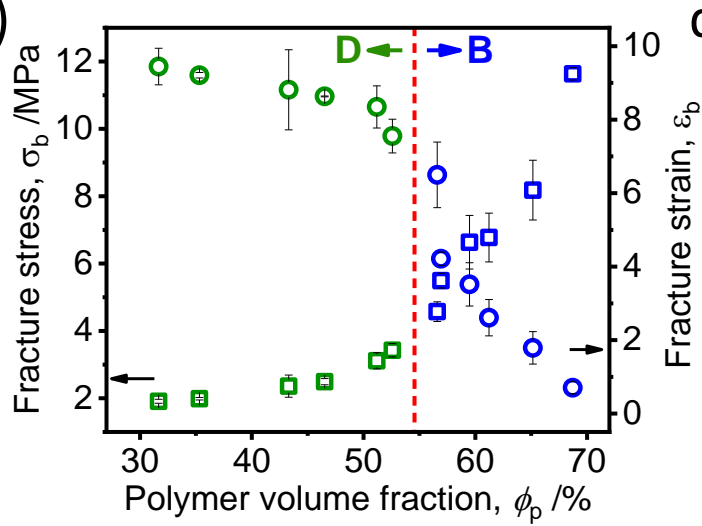

b)

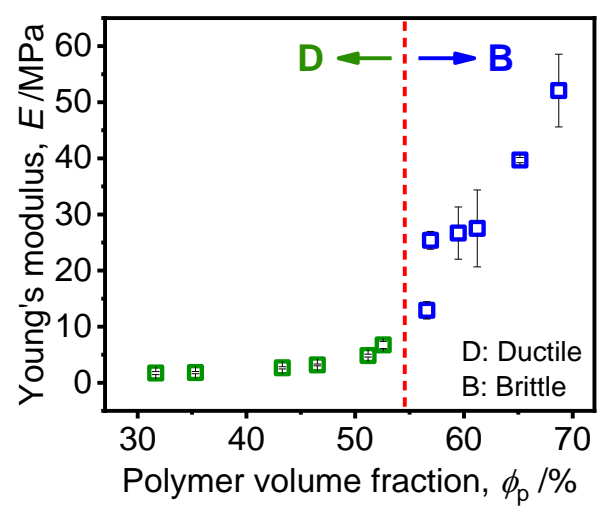

d)

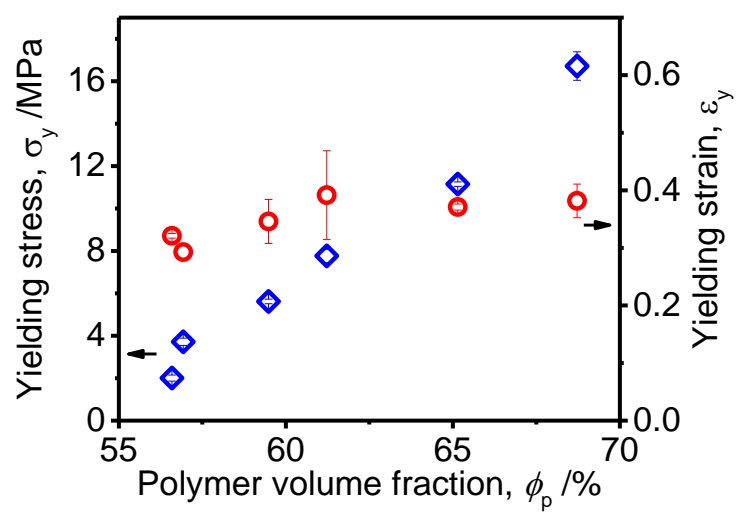

Figure 5. Tensile performance of the PA hydrogels. (a) Stress-strain curves of PA gels at different polymer volume fraction $\phi_{\mathrm{p}}$ under uniaxial tensile test. (b-d) Dependence of (b) Young's modulus $E$, (c) facture stress $\sigma_{\mathrm{b}}$ and fracture strain $\varepsilon_{\mathrm{b}}$, as well as (d) yielding stress $\sigma_{\mathrm{y}}$ and yielding strain $\varepsilon_{\mathrm{y}}$ on $\phi_{\mathrm{p}}$. The tensile test was performed at a nominal strain rate 0.14 $\mathrm{s}^{-1}$.

Figure $5 \mathrm{~b}$ and $5 \mathrm{c}$ summarize the Young's modulus $E$, fracture stress $\sigma_{\mathrm{b}}$ and fracture strain $\varepsilon_{\mathrm{b}}$ at different polymer volume fraction $\phi_{\mathrm{p}}$. For $\phi_{\mathrm{p}} \leq 52.6 \%$, both $E$ and $\sigma_{\mathrm{b}}$ show a gradual increase with $\phi_{\mathrm{p}}$, accompanying with a slightly decrease of $\varepsilon_{\mathrm{b}}$. Once $\phi_{\mathrm{p}}>52.6 \%$, 
both $E$ and $\sigma_{\mathrm{b}}$ increase sharply, while $\varepsilon_{\mathrm{b}}$ decreases drastically with $\phi_{\mathrm{p}}$. For example, varying $\phi_{\mathrm{p}}$ from 31.7 to $52.6 \%$ only leads to $E$ changing from 1.7 to $6.7 \mathrm{MPa}$ and $\varepsilon_{\mathrm{b}}$ changing from 9.4 to 7.6 , while $E$ reaches to $52.1 \mathrm{MPa}$ and $\varepsilon_{\mathrm{b}}$ decreases to 0.7 when further increasing $\phi_{\mathrm{p}}$ to $68.7 \%$. Obviously, the dependencies of stress-strain curves, $E, \sigma_{\mathrm{b}}$, and $\varepsilon_{\mathrm{b}}$ on $\phi_{\mathrm{p}}$ suggest that the mechanical behavior of PA hydrogels changes from viscoelastic to glassy-like by increasing $\phi_{\mathrm{p}}$, and the boundary of viscoelastic-glassy transition is at $\phi_{\mathrm{p}}=52.6 \%$. Figure $5 \mathrm{~d}$ summarizes the yielding stress $\sigma_{\mathrm{y}}$ and yielding strain $\varepsilon_{\mathrm{y}}$ as a function of $\phi_{\mathrm{p}}$, where $\sigma_{\mathrm{y}}$ increases from 2.0 to 16.7 MPa while $\varepsilon_{\mathrm{y}}$ has weak change by increasing $\phi_{\mathrm{p}}$.

The increase in polymer volume fraction $\phi_{\mathrm{p}}$ cause two effects, one is the increase in the density of the original polymer chain and the other is the structure transformation from heterogeneous to homogeneous. For the former effect, by assuming the polymer density change is homogeneous during dehydration, the Young's modulus $E$, fracture stress $\sigma_{\mathrm{b}}$, and fracture strain $\varepsilon_{\mathrm{b}}$ are related to $\phi_{\mathrm{p}}$ by the following equations: $E=E^{N} \phi_{\mathrm{p}}, \sigma_{\mathrm{b}}=\sigma_{\mathrm{b}}^{N} \phi_{\mathrm{p}}^{2 / 3}$ and $\lambda_{\mathrm{b}}=\lambda_{\mathrm{b}}^{N} \phi_{\mathrm{p}}^{1 / 3}$, where $E^{N}, \sigma_{\mathrm{b}}^{N}$ and $\lambda_{\mathrm{b}}^{N}$ are the values at the virtual reference state $\left(\phi_{\mathrm{p}}=1\right){ }^{18}$ Here $\lambda_{\mathrm{b}}=\varepsilon_{\mathrm{b}}+1$ is the fracture stretch ratio. To separate these two effects, we converted $E$, $\sigma_{\mathrm{b}}$, and $\lambda_{\mathrm{b}}$ at various polymer volume fraction $\phi_{\mathrm{p}}$ into the virtual reference state $E^{N}, \sigma_{\mathrm{b}}^{N}$, and $\lambda_{\mathrm{b}}^{N}$, respectively. The results are shown in Figure S4, Supporting Information. For $\phi_{\mathrm{p}} \leq$ $52.6 \%, E^{N}$ and $\sigma_{\mathrm{b}}^{N}$ slightly increase, and $\lambda_{\mathrm{b}}^{N}$ slightly decreases with the increase in $\phi_{\mathrm{p}}$. While for $\phi_{\mathrm{p}}>52.6 \%$, the $E^{N}$ and $\sigma_{\mathrm{b}}^{N}$ increases, and $\lambda_{\mathrm{b}}^{N}$ decreases abruptly, demonstrating that the viscoelastic-glassy transition originated from the structure change rather than increase of polymer density. The yielding stress at the virtual reference state $\left(\phi_{\mathrm{p}}=1\right)$ also 
increases drastically with $\phi_{\mathrm{p}}$ while the yielding deformation ratio at the reference state shows a constant value (Figure S5, Supporting Information). These results also indicate that in high $\phi_{\mathrm{p}}$ region, the increase of $\phi_{\mathrm{p}}$ leads to the PA gels to form glassy materials due to enhanced ionic interaction.

\section{Toughness and self-healing.}

a)

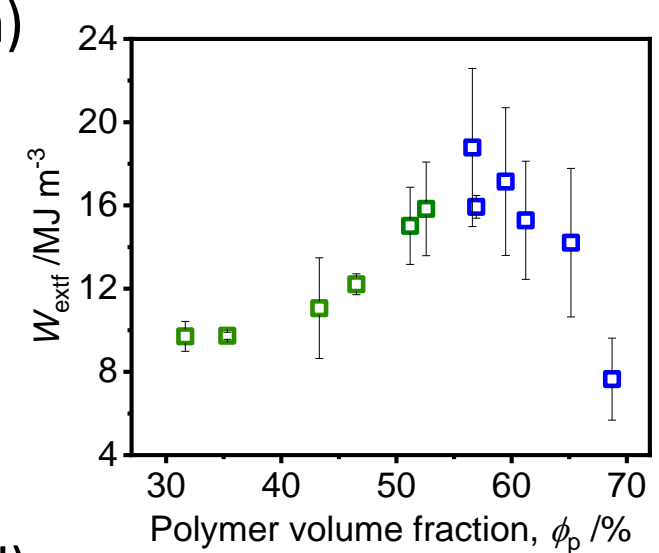

d)

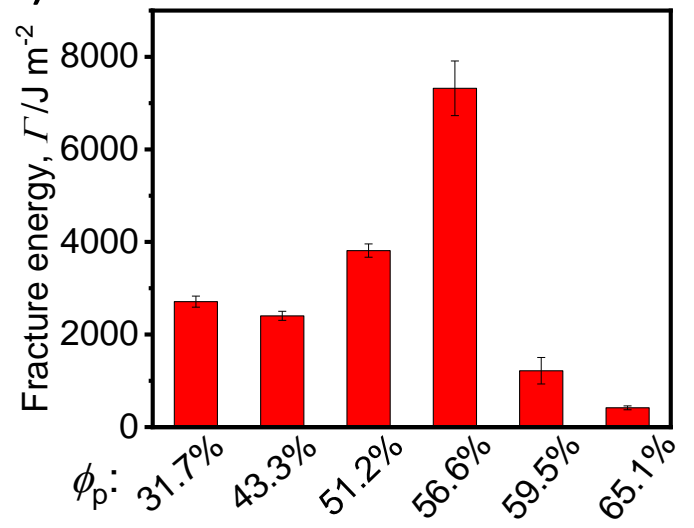

b)

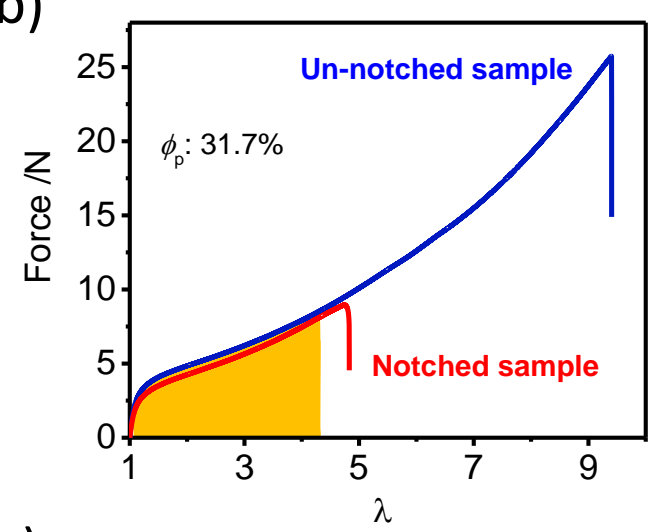

c)

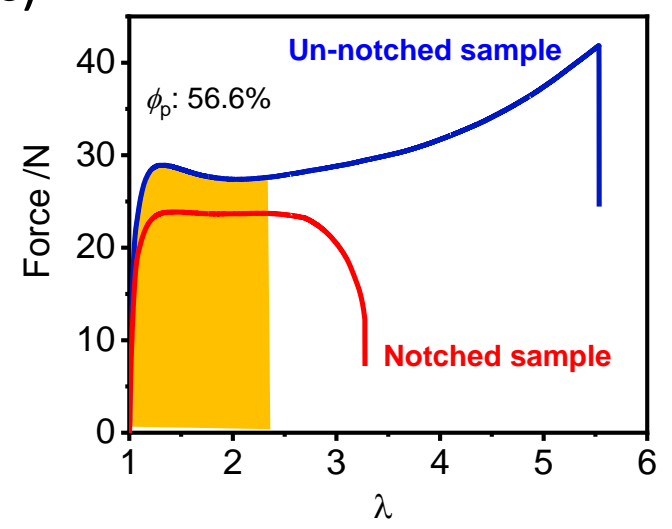

Figure 6. Toughness of PA hydrogels. (a) Work of extension at fracture $W_{\text {extf }}$ at different $\phi_{\mathrm{p}}$. $(b, c)$ Force-stretch ratio $(\lambda)$ curves of notched and un-notched samples at $\phi_{\mathrm{p}}=31.7 \%$ (b) and $\phi_{\mathrm{p}}=56.9 \%$ (c). (d) Fracture energy $\Gamma$ at various $\phi_{\mathrm{p}}$. 
Based on the stress-strain curves in Figure 5, the work of extension at fracture $W_{\text {extf }}$ can be calculated as a parameter to characterize the toughness of PA hydrogels (Figure 6a). $W_{\text {extf }}$ firstly increases with $\phi_{\mathrm{p}}$ and then decreases, giving a maximum around the structural transition point (heterogeneous-homogeneous transition). The maximum of $W_{\text {extf }}$ is $18.8 \mathrm{MJ}$ $\mathrm{m}^{-3}$, almost twice of the reported most tough hydrogels of phase-separation polyacrylamide hydrogel $^{28}$. Single-edge notch test was also performed to evaluate the toughness of PA hydrogels following the previous studies ${ }^{38}$. Figure $6 \mathrm{~b}$ and $6 \mathrm{c}$ show typical force-stretch ratio curves for the un-notched and notched samples at $\phi_{\mathrm{p}}=31.7 \%$ (bicontinuous structure) and $\phi_{\mathrm{p}}=56.6 \%$ (around the structural transition point), respectively. The critical stretch ratio $\lambda_{\mathrm{c}}$ where crack propagation starts for the notched samples decreases with increasing $\phi_{\mathrm{p}}$. For example, $\lambda_{\mathrm{c}}$ for sample with $\phi_{\mathrm{p}}=31.7 \%$ is 4.3 (Figure $6 \mathrm{~b}$ ), while decreases to 2.4 by increasing $\phi_{\mathrm{p}}$ to $56.6 \%$ (Figure $6 \mathrm{c}$ ). The toughness represented by fracture energy $\Gamma$ can be estimated from the yellow area in Figure $6 \mathrm{~b}$ or $6 \mathrm{c}$. The evolution of fracture energy $\Gamma$ with $\phi_{\mathrm{p}}$ is consistent with that of $W_{\text {extf }}$ (Figure 6d). The fracture energy for sample with $\phi_{\mathrm{p}}=$ $31.7 \%$ is about $2700 \mathrm{~J} \mathrm{~m}^{-2}$, in agreement with our previous results ${ }^{21}$, and demonstrates high toughness. The fracture energy of sample at maximum $W_{\text {extf }}$ is about $7300 \mathrm{~J} \mathrm{~m}^{-2}$, which is much larger than the tough double-network gels even with much higher modulus (12.9 MPa). The DN hydrogels known as extremely tough hydrogels possess fracture energy of 100 1000 $\mathrm{Jm}^{-2}$ and modulus of $0.1 \sim 1.0 \mathrm{MPa} .{ }^{16}$ This result also indicates that, by tuning the structure through simple osmotic stress method, one can make PA hydrogels much stronger and tougher. 

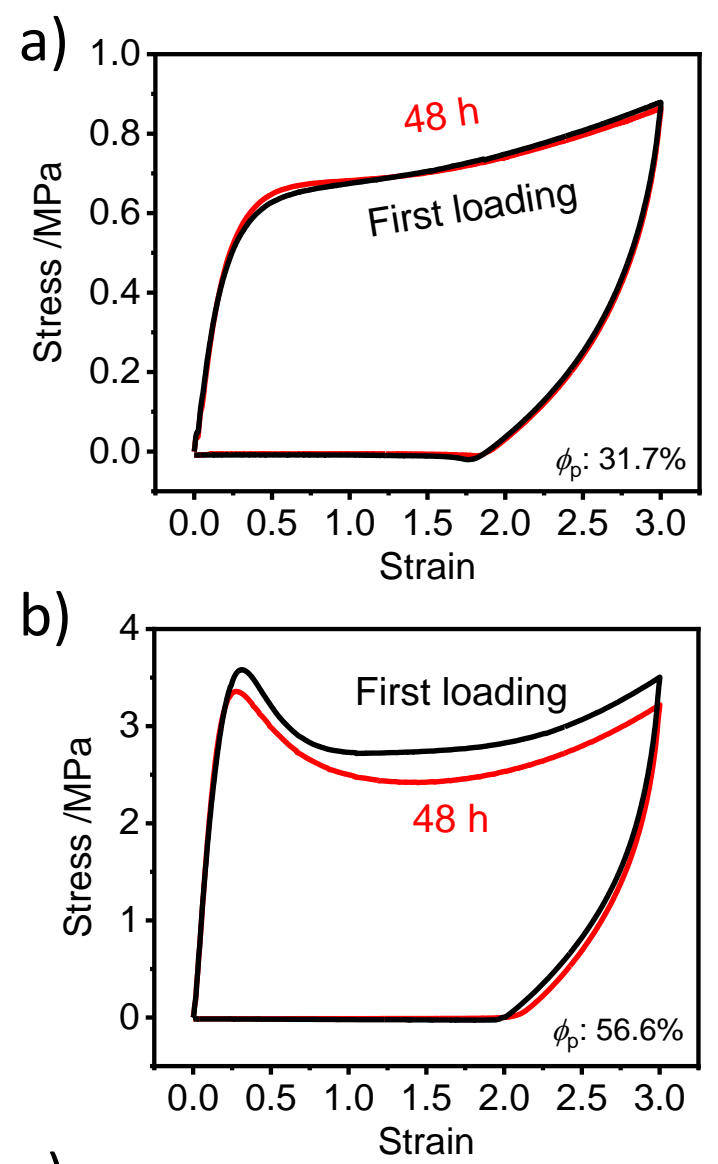

c)

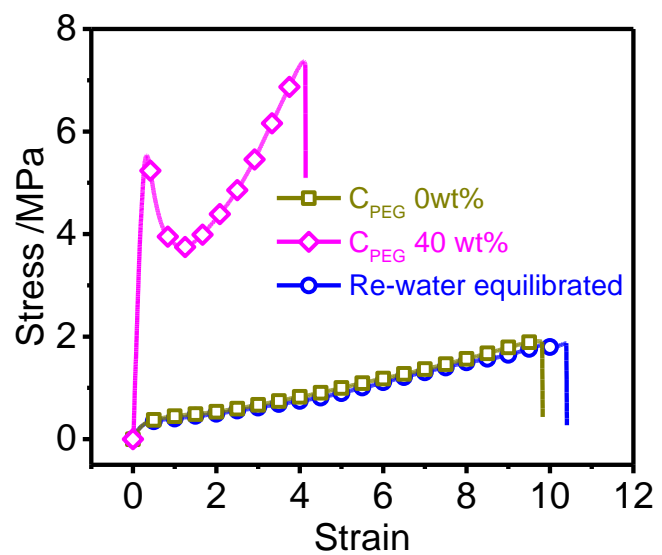

Figure 7. Loading and unloading uniaxial test of PA hydrogels with (a) $\phi_{\mathrm{p}}=31.7 \%$ in water (0 wt $\%$ PEG solution) and (b) $\phi_{\mathrm{p}}=56.6 \%$ in $30 \mathrm{wt} \%$ PEG solution. (c) Stress-strain curves of water-equilibrated sample, $40 \mathrm{wt} \%$ PEO solution-equilibrated sample $\left(\phi_{\mathrm{p}}=59.5 \%\right)$ as well as re-water-equilibrated sample. The stretch speed was $100 \mathrm{~mm} / \mathrm{min}$. 
Since the broken physical ionic interactions are able to reform, the PA hydrogels possess self-recovery ability. As revealed in our previous study ${ }^{30}$, the gel with bicontinuous structure $\left(\phi_{\mathrm{p}}=31.7 \%\right.$, in water) shows fully self-recovery before the internal fracture of the rigid phase that occurs above a strain of 3.0. Here we compare the self- recovery behaviors of two representative samples, the sample with bicontinuous structure $\left(\phi_{\mathrm{p}}=31.7 \%\right.$, in water $)$ and the sample with homogeneous structure ( $\phi_{\mathrm{p}}=56.6 \%$ in $30 \mathrm{wt} \%$ PEG solution), which are shown by the cyclic loading-unloading stress-strain curves in Figure $7 \mathrm{a}$ and $7 \mathrm{~b}$, respectively. The maximum strain is set at 3.0, at which the sample with bicontinuous structure shows the full self-recovery. A large mechanical hysteresis is observed in both two samples, indicating large energy dissipation during deformation that is responsible for the high toughness of the hydrogels. The loading-unloading test was repeated after $48 \mathrm{~h}$ for those two samples without any thermal or chemical processing. In contrast to the sample with bicontinuous structure that shows fully recovery in its stress-strain curve, the homogeneous sample shows only $92 \%$ self-recovery in the hysteresis area although the residual strain fully disappears after the long waiting time (Figure $7 b$ ). The latter result indicates that some covalent bonds are broken in the case of the rigid homogeneous sample. It is interesting that although this sample shows a prominent yielding, during which the strong physical associations break, most of them recovered after enough waiting. This behavior is significantly different with the chemical DN gels, in which the yielding is due to the scission of covalent bonds in the first network ${ }^{16}$. The maximum strain to show full self-recovery decreases with the increase of the polymer fraction $\phi_{\mathrm{p}}$, indicating that the decrease of water 
content freezes the dynamics of the ionic bonds and leads to partial damage of the covalent bonds in the case of PA gels above the viscoelastic-glassy transition point.

The structural transition induced by osmotic stress is reversible. When the hydrogels with a homogeneous structure was immersed into water to re-equilibrate, the homogeneous structure transform into bicontinuous structure and the gels recover to their initial sizes and mechanical properties. Figure 7c presents an example to demonstrate this property. The sample equilibrated in PEO solution $\left(C_{\mathrm{PEG}}=40 w t \%, \phi_{\mathrm{p}}=59.5 \%\right)$ shows glassy like behavior with obvious yielding, high modulus, large fracture stress but small fracture strain. After immersing in water to re-equilibrate, this sample becomes viscoelastic and ductile, which is almost the same as the originally water-equilibrated sample $\left(C_{\mathrm{PEG}}=0 \mathrm{wt} \%\right)$. This result demonstrates that the transition of mechanical property is due to structure change but without change of the topological chain network of gel.

\section{Structure-property relationship.}

Based on above results, we can make a brief summary here. (i) The osmotic stress leads to condensation of polymer and thus enhanced physical ionic interaction of PA hydrogels. (ii) USAXS and SAXS results suggest that the bicontinuous structure with structural length of about $400 \mathrm{~nm}$ transforms into homogeneous structure by increasing osmotic stress or polymer volume fraction $\phi_{\mathrm{p}}$. (iii) With increasing $\phi_{\mathrm{p}}$, optical results indicate an opaque-transparent transition, and mechanical results present a viscoelasticglassy transition. (iv) The gels near the structural and mechanical transition point possess

maximum toughness with work of extension at fracture of $18.8 \mathrm{MJ} \mathrm{m}^{-3}$ and fracture energy of $7200 \mathrm{~J} \mathrm{~m}^{-2}$. (v) Both bicontinuous gel and homogeneous gel in this study show almost 
fully self-recovery in a certain strain range even after obvious yielding and the structural transition induced by osmotic stress is reversible. It should be noted that the heterogeneoushomogeneous transition in structure corresponds to transitions in optical and mechanical properties exactly, suggesting the intrinsic correlation between structure and properties of PA hydrogels.

The structure of PA hydrogels is heterogeneous with local polymer dense region (hard region) and sparse region (soft region) for $\phi_{\mathrm{p}} \leq 52.6 \%$ (Figure $4 \mathrm{a}$ ). Up to a relatively large deformation, the bicontinuous network shows a reversible affine deformation, during which nanoscale chain conformations change with ionic bond breaking in the two regions dissipating energy. At larger deformations, the bicontinuous network is highly stretched, and some parts of hard network start to rupture as they carry most of the stress, while the soft network can decrease stress concentration and transfer stress via shear. Such a meso-scale double network structure prevents the catastrophic propagation of hard phase rupture, leading to a large deformability of the gels before global failure. Consequently, the PA gels with bicontinuous structure are tough and highly viscoelastic. ${ }^{30}$

When the osmotic stress or $\phi_{\mathrm{p}}$ larger than a threshold $\left(\phi_{\mathrm{p}}=52.6 \%\right.$ for PA in this work), the polymer sparse region becomes dense enough as polymer dense region to form a homogeneous structure (Figure 4c). As a result, there is no soft network to transfer stress and decrease stress concentration. Thereby the sample is easy to fracture once microcracks generate, as demonstrated by the small fracture strain of the gels with a homogeneous structure (Figure 5c). At this stage, increasing $\phi_{\mathrm{p}}$ leads to the homogeneous structure to form much stronger ionic interaction as crosslinking point, as the ionic interaction is polymer 
concentration dependent according to electrostatic interaction theory. ${ }^{21,33}$ This explains why the Young's modulus and fracture stress increase sharply with $\phi_{\mathrm{p}}$ (Figure 5).

The maximum toughness of PA hydrogels near the structural transition point is the result of two opposite effects. The increase of the ionic bond strength that relevant for the strength of gel and the decrease of the relative strength of soft network and hard network that relevant for deformability. For the bicontinuous structure gels (Figure 4a), the existence of soft network can effectively transfer stress and reduce stress concentration, endowing the gel a large deformability, during which the breaking of ionic bonds and the rupture of hard network dissipate energy. However, the strength of ionic bonds at this stage is relatively weak, meaning that less energy is dissipated by breaking ionic bonds during deformation. When the soft network becomes the same as the hard network by dehydration (the gel becomes homogeneous, Figure 4c), the stress-transferring and crack-stopping mechanisms disappear due the absence of soft network, resulting in a small deformability and thus low toughness of gels. While around the structural transition point (Figure 4b), the soft network is comparable, but "weaker" than the hard network. At this stage, the soft network still can transfer stress and reduce stress concentration to give a large deformability of the gels. At the same time, the ionic bonds are relatively strong to dissipate significant amount of energy. As a result, the gels around the structural transition point possess maximum toughness.

One may argue whether the sharp decrease in deformability is due to structure change from heterogeneous to homogeneous, or solely due to the modulus change by dehydrating gels into glassy-like state. To see the effect of heterogeneous structure, we compared the fracture strain of bicontinuous and homogeneous samples at the same modulus. The gel with 
$\phi_{\mathrm{p}}=31.7 \%$ was chosen to represent the bicontinuous sample, and the gel with $\phi_{\mathrm{p}}=59.5 \%$ was chosen to represent the homogeneous sample. To compare their mechanical performance at the same modulus, the gel with $\phi_{\mathrm{p}}=31.7 \%$ was tested at room temperature, while the gel with $\phi_{\mathrm{p}}=59.5 \%$ was tested at $52{ }^{\circ} \mathrm{C}$, at the same nominal strain rate of $0.14 \mathrm{~s}^{-1}$. The $\phi_{\mathrm{p}}$ of gel decreases slightly from $59.5 \%$ at room temperature to $56.5 \%$ at $52{ }^{\circ} \mathrm{C}$ due to thermal expansion. Figure 8a shows the stress-strain curves of these two samples. The sample with homogeneous structure has a higher stress but lower extensibility than the sample with bicontinuous structure. Figure $8 \mathrm{~b}$ shows the corresponding normalized Young's modulus and normalized fracture strain after removing polymer density effect with aforementioned method. Although their normalized Young's moduli are almost same, the gel with bicontinuous structure is more stretchable than the gel with homogeneous structure, suggesting that the bicontinuous structure results in delayed fracture due to the meso-scale double network effect. ${ }^{30}$ It should be noted that a different batch of gels was used in this test, and this batch of samples were slightly more stretchable than those used in the previous tests of this work.

a)

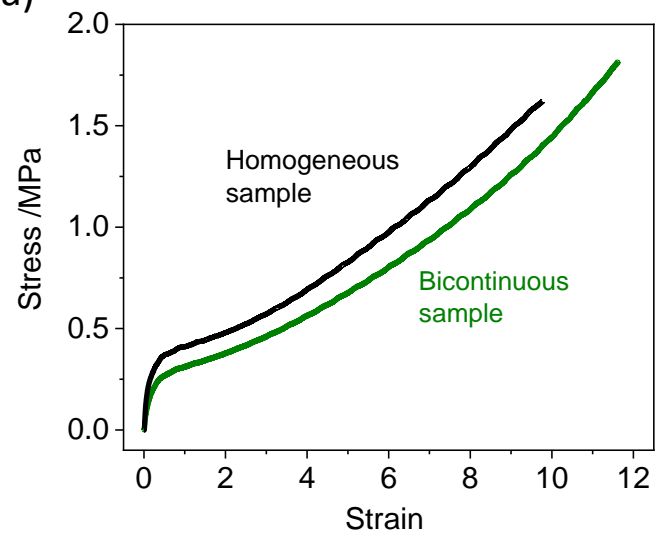

b)

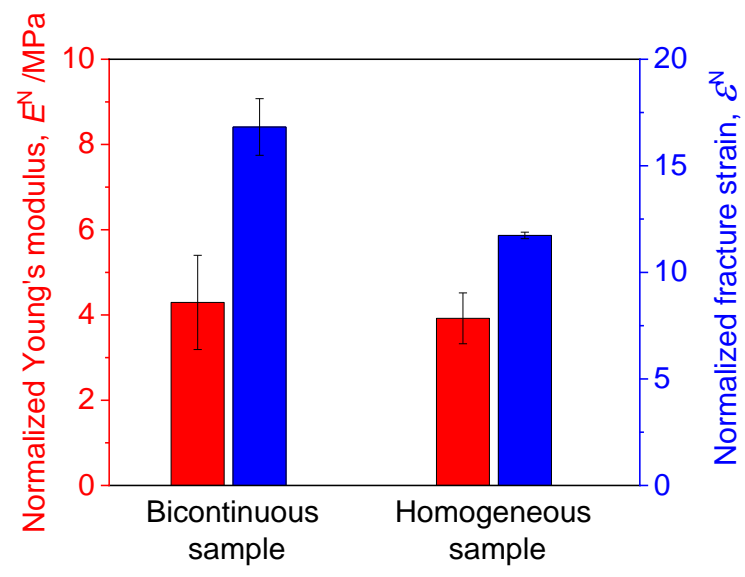


Figure 8. (a) Uniaxial tensile stress-strain curves of PA gels with bicontinuous structure $\left(\phi_{\mathrm{p}}=31.7 \%\right)$ measured at room temperature and with homogeneous structure $\left(\phi_{\mathrm{p}}=56.5 \%\right)$ measured at $52{ }^{\circ} \mathrm{C}$. (b) The corresponding normalized Young's modulus $E^{\mathrm{N}}$ and normalized fracture strain $\mathcal{E}^{\mathrm{N}}$. The test was performed at a nominal strain rate $0.14 \mathrm{~s}^{-1}$.

\section{CONCLUSION}

In conclusion, by adopting osmotic stress method, we elucidated the missing link between structure and properties of PA hydrogels, and demonstrated that the optical and mechanical properties can be tuned in a wide range by changing ionic interaction. Specifically, 1) increasing osmotic stress of the bath solution triggers the dehydration of gel and resultant enhanced ionic interaction; 2) the mesoscale bicontinuous network structure transforms into homogeneous structure due to the difference in dehydration ability of the hard network and the soft network; 3) the heterogeneous-homogeneous transitions in structure drives concurrently opaque-transparent transition in optical property, and viscoelastic-glassy transition in mechanical property; 4) gels near the structural transition point was found to possess maximum toughness (fracture energy $7200 \mathrm{~J} \mathrm{~m}^{-2}$ ), high stiffness (Young's modulus of $12.9 \mathrm{MPa}$ ) and self-recoverable ability (92\% self-recovery); 5) the maximum toughness is due to the balance between enhanced ionic bond strength and decreased deformability by structure homogenization. This work not only establishes an approach to investigate the effect of structure-property relationship of physical hydrogels, but also gives inspiration to design tough and self-healing network materials around the structure transition point.

\section{ACKNOWLEDGES}


This research was partially supported by Postdoctoral Fellowships for Foreign Researchers (Grant No. 15F15348) from the Japan Society for the Promotion of Science (JSPS) and JSPS KAKENHI (Grant No. JP17H06144). The Institute for Chemical Reaction Design and Discovery (ICReDD) was established by World Premier International Research Initiative (WPI), MEXT, Japan. The USAXS measurement was conducted at BL20XU beamline of Spring-8 (JASRI, Japan) and the SAXS was performed in BL16B beamline of Shanghai Synchrotron Radiation Facility (SSRF, China).

\section{ASSOCIATED CONTENT}

\section{Supporting Information}

The Supporting Information is available free of charge on the ACS Publications website at DOI:

Figure S1 gives the water content and transmittance for PA hydrogels re-equilibrated at different PEO aqueous solutions.

Figure S2 gives overlaying USAXS and SAXS profiles of PA hydrogels.

Figure S3 presents linear dynamic behavior of PA hydrogels.

Figure S4 presents the Young's modulus, fracture stress and fracture stretch ratio at the virtual reference state $\left(\phi_{\mathrm{p}}=1\right)$.

Figure S5 gives the yielding stress and yielding strain at the virtual reference state $\left(\phi_{\mathrm{p}}=1\right)$. 


\section{REFERENCES}

(1) Guo, M.; Pitet, L. M.; Wyss, H. M.; Vos, M.; Dankers, P. Y. W.; Meijer, E. W. Tough Stimuli-Responsive Supramolecular Hydrogels with Hydrogen-Bonding Network Junctions. J. Am. Chem. Soc. 2014, 136 (19), 6969-6977.

(2) Tanaka, T.; Sato, E.; Hirokawa, Y.; Hirotsu, S.; Peetermans, J. Critical Kinetics of Volume Phase Transition of Gels. Phys. Rev. Lett. 1985, 55 (22), 2455-2458.

(3) Gong, J. P.; Hirota, N.; Kakugo, A.; Narita, T.; Osada, Y. Effect of Aspect Ratio on Protein Diffusion in Hydrogels. J. Phys. Chem. B 2000, 104, 9904-9908.

(4) Hoare, T. R.; Kohane, D. S. Hydrogels in Drug Delivery: Progress and Challenges. Polymer. 2008, pp 1993-2007.

(5) Kamath, K. R.; Park, K. Biodegradable Hydrogels in Drug Delivery. Adv. Drug Deliv. Rev. 1993, 11 (1-2), 59-84.

(6) Lee, K. Y.; Mooney, D. J. Hydrogels for Tissue Engineering. Chemical Reviews. 2001, pp 1869-1879.

(7) Shibayama, M. Spatial Inhomogeneity and Dynamic Fluctuations of Polymer Gels. Macromol. Chem. Phys. 1998, 199 (1), 1-30.

(8) Bonn, D.; Kellay, H.; Prochnow, M.; Ben-Djemiaa, K.; Meunier, J. Delayed Fracture Of An Inhomogeneous Soft Solid. Science (80-. ). 1998, 280 (5361), 265-267.

(9) Tanaka, Y.; Fukao, K.; Miyamoto, Y. Fracture Energy of Gels. Eur. Phys. J. E. Soft 
Matter 2000, 401 (3), 395-401.

(10) Okumura, Y.; Ito, K. The Polyrotaxane Gel: A Topological Gel by Figure-of-Eight Cross-Links. Adv. Mater. 2001, 13 (7), 485-487.

(11) Haraguchi, K.; Takehisa, T. Nanocomposite Hydrogels: A Unique Organic-Inorganic Network Structure with Extraordinary Mechanical, Optical, and Swelling/DeSwelling Properties. Adv. Mater. 2002, 14 (16), 1120-1124.

(12) Malkoch, M.; Vestberg, R.; Gupta, N.; Mespouille, L.; Dubois, P.; Mason, A. F.; Hedrick, J. L.; Liao, Q.; Frank, C. W.; Kingsbury, K.; Hawker, C. J. Synthesis of Well-Defined Hydrogel Networks Using Click Chemistry. Chem. Commun. 2006, No. 26, 2774-2776.

(13) Ossipov, D. A. Poly ( Vinyl Alcohol ) -Based Hydrogels Formed by “ Click Chemistry ." 2006, No. i, 1709-1718.

(14) Sakai, T.; Matsunaga, T.; Yamamoto, Y.; Ito, C.; Yoshida, R.; Suzuki, S.; Sasaki, N.; Shibayama, M.; Chung, U. Il. Design and Fabrication of a High-Strength Hydrogel with Ideally Homogeneous Network Structure from Tetrahedron-like Macromonomers. Macromolecules 2008, 41 (14), 5379-5384.

(15) Ahmed, S.; Nakajima, T.; Kurokawa, T.; Anamul Haque, M.; Gong, J. P. Brittleductile Transition of Double Network Hydrogels: Mechanical Balance of Two Networks as the Key Factor. Polymer (Guildf). 2014, 55 (3), 914-923.

(16) Gong, J. P. Why Are Double Network Hydrogels so Tough? Soft Matter 2010, 6 
(12), 2583-2590.

(17) Haque, M. A.; Kurokawa, T.; Gong, J. P. Super Tough Double Network Hydrogels and Their Application as Biomaterials. Polymer (Guildf). 2012, 53 (9), 1805-1822.

(18) Itagaki, H.; Kurokawa, T.; Furukawa, H.; Nakajima, T.; Katsumoto, Y.; Gong, J. P. Water-Induced Brittle-Ductile Transition of Double Network Hydrogels.

Macromolecules 2010, 43 (22), 9495-9500.

(19) Gong, J. P.; Katsuyama, Y.; Kurokawa, T.; Osada, Y. Double-Network Hydrogels with Extremely High Mechanical Strength. Adv. Mater. 2003, 15 (14), 1155-1158.

(20) Sun, J.-Y.; Zhao, X.; Illeperuma, W. R. K.; Chaudhuri, O.; Oh, K. H.; Mooney, D. J.; Vlassak, J. J.; Suo, Z. Highly Stretchable and Tough Hydrogels. Nature 2012, 489 (7414), 133-136.

(21) Sun, T. L.; Kurokawa, T.; Kuroda, S.; Ihsan, A. Bin; Akasaki, T.; Sato, K.; Haque, M. A.; Nakajima, T.; Gong, J. P. Physical Hydrogels Composed of Polyampholytes Demonstrate High Toughness and Viscoelasticity. Nat. Mater. 2013, 12 (10), 932937.

(22) Narita, T.; Mayumi, K.; Ducouret, G.; Hébraud, P. Viscoelastic Properties of Poly(Vinyl Alcohol) Hydrogels Having Permanent and Transient Cross-Links Studied by Microrheology, Classical Rheometry, and Dynamic Light Scattering. Macromolecules 2013, 46 (10), 4174-4183.

(23) Luo, F.; Sun, T. L.; Nakajima, T.; Kurokawa, T.; Zhao, Y.; Sato, K.; Ihsan, A. B.; Li, 
X.; Guo, H.; Gong, J. P. Oppositely Charged Polyelectrolytes Form Tough, SelfHealing, and Rebuildable Hydrogels. Adv Mater 2015, 27 (17), 2722-2727.

(24) Okay, O. Self-Healing Hydrogels Formed via Hydrophobic Interactions. Supramol. Polym. Networks Gels 2015, 268, 101-142.

(25) Zhang, H. J.; Sun, T. L.; Zhang, A. K.; Ikura, Y.; Nakajima, T.; Nonoyama, T.; Kurokawa, T.; Ito, O.; Ishitobi, H.; Gong, J. P. Tough Physical Double-Network Hydrogels Based on Amphiphilic Triblock Copolymers. Adv. Mater. 2016, 28 (24), 4884-4890.

(26) Ihsan, A. Bin; Sun, T. L.; Kuroda, S.; Haque, M. A.; Kurokawa, T.; Nakajima, T.; Gong, J. P. A Phase Diagram of Neutral Polyampholyte - from Solution to Tough Hydrogel. J. Mater. Chem. B 2013, 1 (36), 4555.

(27) Ihsan, A. Bin; Sun, T. L.; Kurokawa, T.; Karobi, S. N.; Nakajima, T.; Nonoyama, T.; Roy, C. K.; Luo, F.; Gong, J. P. Self-Healing Behaviors of Tough Polyampholyte Hydrogels. Macromolecules 2016, 49 (11), 4245-4252.

(28) Sato, K.; Nakajima, T.; Hisamatsu, T.; Nonoyama, T.; Kurokawa, T.; Gong, J. P. Phase-Separation-Induced Anomalous Stiffening, Toughening, and Self-Healing of Polyacrylamide Gels. Adv. Mater. 2015, 27 (43), 6990-6998.

(29) Guo, H.; Sanson, N.; Hourdet, D.; Marcellan, A. Thermoresponsive Toughening with Crack Bifurcation in Phase-Separated Hydrogels under Isochoric Conditions. Adv. Mater. 2016, 28 (28), 5857-5864. 
(30) Cui, K.; Sun, T. L.; Liang, X.; Nakajima, K.; Ye, Y. N.; Chen, L.; Kurokawa, T.;

Gong, J. P. Multiscale Energy Dissipation Mechanism in Tough and Self-Healing Hydrogels. Phys. Rev. Lett. 2018, 121 (18), 185501.

(31) Money, N. P. Osmotic Pressure of Aqueous Polyethylene Glycols 1 Relationship between Molecular Weight and Vapor Pressure Deficit. Plant Physiol. 1989, 91 (2), $766-769$.

(32) Delage, P.; Howat, M. D.; Cui, Y. J. The Relationship between Suction an Welling Properties in Heaving Compacted Unsaturated Clay. Eng. Geol. 1998, 50, 31-48.

(33) English, A. E.; Mafé, S.; Manzanares, J. a.; Yu, X.; Grosberg, A. Y.; Tanaka, T. Equilibrium Swelling Properties of Polyampholytic Hydrogels. J. Chem. Phys. 1996, $104(21), 8713$.

(34) Cui, K.; Sun, T. L.; Kurokawa, T.; Nakajima, T.; Nonoyama, T.; Chen, L.; Gong, J. P. Stretching-Induced Ion Complexation in Physical Polyampholyte Hydrogels. Soft Matter 2016, 12 (43), 8833-8840.

(35) Sun, T. L.; Luo, F.; Kurokawa, T.; Karobi, S. N.; Nakajima, T.; Gong, J. P. Molecular Structure of Self-Healing Polyampholyte Hydrogels Analyzed from Tensile Behaviors. Soft Matter 2015, 11 (48), 9355-9366.

(36) Hammersley, A. P. FIT2D: An Introduction and Overview. ESRF Intern. Report, ESRF97HAO2T 1997.

(37) Greensmith, H. W. Rupture of Rubber. X. The Change in Stored Energy on Making 
a Small Cut in a Test Piece Held in Simple Extension. J. Appl. Polym. Sci. 1963, 7 (3), 993-1002.

(38) Mayumi, K.; Guo, J.; Narita, T.; Hui, C. Y.; Creton, C. Fracture of Dual Crosslink Gels with Permanent and Transient Crosslinks. Extrem. Mech. Lett. 2016, 6, 52-59.

(39) Eisei, T.; Liu, A.; Nakada, T. Edible Eyeballs from Fish. Nature. 1990, pp 298-299.

(40) Li, X.; Charaya, H.; Bernard, G. M.; Elliott, J. A. W.; Michaelis, V. K.; Lee, B.; Chung, H. J. Lowerature Ionic Conductivity Enhanced by Disrupted Ice Formation in Polyampholyte Hydrogels. Macromolecules 2018, 51 (7), 2723-2731.

(41) Boldon, L.; Laliberte, F.; Liu, L. Review of the Fundamental Theories behind Small Angle X-Ray Scattering, Molecular Dynamics Simulations, and Relevant Integrated Application. Nano Rev. 2015, 6, 25661.

(42) Bauwens-Crowet, C.; Bauwens, J. C.; Homès, G. Tensile Yield-Stress Behavior of Glassy Polymers. J. Polym. Sci. 1969, 7 (4), 735--742.

(43) Liu, Y.; Truss, R. W. A Study of Tensile Yielding of Isotactic Polypropylene. J. Polym. Sci. Part B Polym. Phys. 1994, 32 (12), 2037-2047. 


\section{For Table of Contents Use Only}

\section{Effect of Structure Heterogeneity on Mechanical Performance of Physical Polyampholytes Hydrogels}

Kunpeng Cui ${ }^{1}$, Ya Nan $\mathrm{Ye}^{2,3}$, Tao Lin Sun ${ }^{2,3,4}$, Liang Chen ${ }^{5}$, Xueyu $\mathrm{Li}^{3}$, Takayuki Kurokawa $^{2,3}$, Tasuku Nakajima ${ }^{1,2,3}$, Takayuki Nonoyama ${ }^{2,3}$, Jian Ping Gong, , $^{1,2 *}$

${ }^{1}$ Institute for Chemical Reaction Design and Discovery (WPI-ICRD), Hokkaido University, Sapporo 001-0021, Japan

${ }^{2}$ Faculty of Advanced Life Science, Hokkaido University, Sapporo 060-0810, Japan

${ }^{3}$ Soft Matter GI-CoRE, Hokkaido University, Sapporo 001-0021, Japan

${ }^{4}$ South China Advanced Institute for Soft Matter Science and Technology, South China University of Technology, Guangzhou 510640, China

${ }^{5}$ Graduate School of Life Science, Hokkaido University, Sapporo 060-0810, Japan

*Corresponding author: gong@sci.hokudai.ac.jp

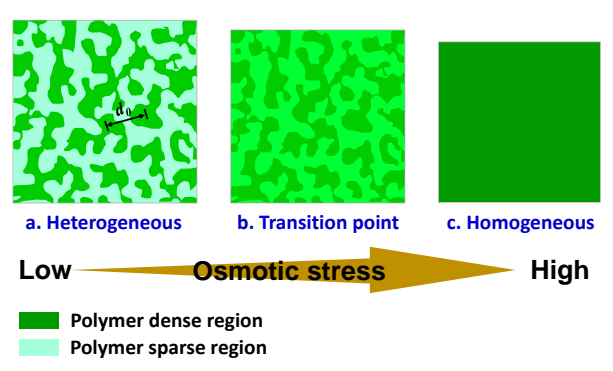

